\title{
MEAN TEMPERATURE PROFILE AT THE ENTRACE OF A THERMOACOUSTIC STACKED SCREEN HEAT EXCHANGER
}

\author{
BÜHLER ${ }^{1}$ Simon, WILCOX ${ }^{2}$ Douglas, OOSTERHUIS ${ }^{1}$ Joris P.,VAN DER MEER ${ }^{1}$ \\ Theo H. \\ Affiliation: ${ }^{1}$ Thermal Engineering, University of Twente, P.O. Box 217 - 7500AE \\ Enschede - The Netherlands, ${ }^{2}$ Chart Inc.-Qdrive, Troy, New York 12180 - USA \\ e-mail address of corresponding author: s.buhler@ utwente.nl
}

\begin{abstract}
The entrance effects at a stacked screen heat exchanger are investigated with two CFD test cases. The first CFD test case models an ideal heat exchanger adjacent to an open space. The influence of the heat conduction on the mean temperature is shown. The second test case models two screens of a stacked screen regenerator as two inline cylinders. The mean temperature profile is compared to the numerical solution of a reduced model equation. It is shown that viscous effects do not influence the mean temperature profile at low amplitude.
\end{abstract}

\section{Introduction}

Thermoacoustic refrigerators pump heat from a cold heat exchanger to an ambient heat exchanger while consuming acoustic power. The thermoacoustic heat pumping occurs in the regenerator, which is situated between two heat exchangers. On the other side of the cold heat exchanger a thermal buffer tube is placed in order to insulate the cold heat exchanger from the secondary ambient heat exchanger, while transferring the acoustic power [1]. Storch et al. [2] reported a distorted temperature profile within the thermal buffer tube, which does not follow the linear thermoacoustic theory derived by Rott [3] and reviewed by Swift [4]. This is due to the violation of the assumption that the displacement amplitude $\xi_{1}$ is much smaller than all other relevant dimensions in the wave propagation direction. In common thermoacoustic refrigerators the length of the heat exchanger is comparable with the displacement amplitude $\xi_{1}$ [4]. In this case the convective effects $(u \cdot \nabla) T$ at the entrance of the heat exchangers are not negligible as they lead to a change in mean temperature, which can be on the order of the adiabatic temperature oscillation. This nonlinear effect leads to an increase in thermal losses, as a steeper temperature gradient occurs in the thermal buffer tube [2].

The change in mean temperature was qualitatively explained by Swift [4] and Kittel et al. [5] by following gas parcels which start within two displacements amplitudes of the entrance of a heat exchanger in the Lagrangian point of view. Summing at one position in the Eulerian point of view the temperature of the gas parcels, the mean temperature profile close to the entrance of the heat exchanger can be obtained. This leads to a joining condition in the mean temperature which is widely accepted and implemented in one-dimensional codes like DeltaEC [6].

Analytical solutions were derived by Matveev et al. [7] and Gusev et al. [8] for the simplified case in which both the heat conduction in the wave propagation direction as well as the viscous effects are neglected.

Next to the analytical solution, also numerical models that include heat conduction in the wave propagation direction were presented by Matveev et al. [9] and Berson et al. [10]. These results were compared to experimental results and showed good agreement, but still left some questions open [9]. 
In this paper the results of a numerical model similar to the one presented in Matveev et al. [9] and Berson et al. [10] is compared to computational fluid dynamics (CFD) simulations. Two CFD models are presented and compared to the simplified models.

\section{Method}

In this paper two CFD models and two simplified models are applied in order to estimate the mean temperature profile close to the heat exchanger in a thermoacoustic heat pump. The CFD models are based on the commercial finite volume code ANSYS Fluent 14 [11]. The working fluid is helium at a mean pressure of $p_{0}=1 \mathrm{~atm}$ and at a temprature of $T_{0}=300 \mathrm{~K}$. In total five periods are simulated and the mean temperature is calculated by averaging the last period. In both models a traveling wave with a frequency of $f=100 \mathrm{~Hz}$ is modeled. The wave enters at the left side of the domain and leaves it at the right, through a non-reflecting boundary.

\section{CFD models}

The first test case consists of an ideal heat exchanger, with an open area. The model and the boundary conditions are presented in Figure 1. The ideal heat exchanger at the left of the domain is modeled with help of a dedicated acoustic boundary condition implemented via a Fluent User Defined Function (UDF), which is similar to the one described in Liao [12]. This boundary condition imposes the pressure at the boundary such that a traveling wave is introduced at the left of the domain with a pressure amplitude of $p_{1}=100 \mathrm{~Pa}$. This wave travels through the computational domain and exits at the right, through another nonreflecting boundary condition. The two acoustic boundary conditions differ in the way the temperature of the incoming fluid is calculated. At the left boundary it is assumed that the incoming fluid is isothermal, in order to model the ideal heat exchanger. At the right boundary the temperature of the incoming fluid is calculated from the pressure assuming adiabatic wave propagation. The horizontal boundary conditions are set to be periodic. The total domain is five displacement amplitudes $\xi_{1}$ long and 0.04 displacement amplitudes $\xi_{1}$ high. The domain is discretized by 100 elements per displacement amplitude in both spatial directions.

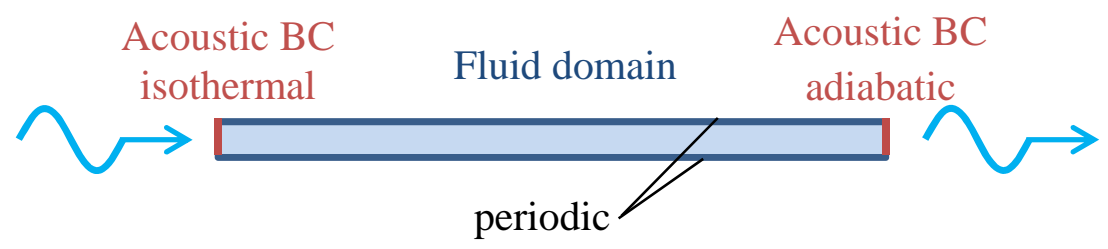

Figure 1: Boundary conditions of the thermal entrance effects model in case of an ideal heat exchanger.

The second case models the entrance effects for a simplified model of a stacked screen heat exchanger. As the main focus is on the entrance effects, only two screens are taken into account. The screens are modeled as an array of cylinders, reducing the problem to two space dimensions. Using periodic boundary conditions the domain can be simplified as shown in Figure 2. At the left and right of the domain the acoustic boundary conditions are applied such that an acoustic wave enters the domain at the left and exits at the right without reflection. The incoming wave has a pressure amplitude of $p_{1}=250 \mathrm{~Pa}$. The temperature of the incoming fluid is calculated from the pressure assuming adiabatic wave propagation. The screens are assumed isothermal and a no-slip velocity boundary is imposed. The radius of the cylinders is $R=0.046 \cdot \xi_{1}$ and the centre of the cylinders are separated by $6 \cdot R$. The total domain is $L_{x}=14.5 \cdot \xi_{1}$ long and $L_{y}=10 \cdot R$ high. 


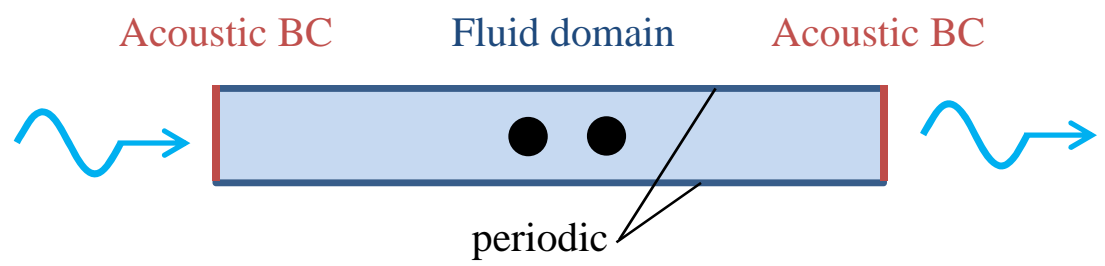

Figure 2: Boundary conditions of the stacked screen heat exchanger test case.

\section{Simplified models}

The aforementioned CFD models are compared against two simplified models. The first is the analytical solution derived by Matveev et al. [7] and the main assumptions for the derivation are:

- No viscous wall effects occur, one dimensional acoustics.

- No heat conduction besides the temperature gradient imposed by the heat exchangers.

- The pressure is spatially constant.

In the CFD models presented above a traveling wave is investigated with no temperature gradient. In this case the analytical solution for the mean temperature can be written as [7]:

$$
T_{m}(x)=T_{0}-\frac{2}{\pi}\left(1-\left(\frac{x}{2 \xi_{1}}\right)^{\frac{\pi}{2}}\right) \frac{\gamma-1}{\gamma} \frac{p_{1}}{p_{0}} T_{0}
$$

The second simplified model solves the temperature equation numerically. While Matveev et al. [9] solved the temperature equation in the Lagrangian point of view, Berson et al. [10] solved the dimensionless temperature equation in the Eulerian coordinates. In this paper the second point of view is chosen and the following temperature equation is solved:

$$
\frac{\partial T}{\partial t}=\frac{\gamma-1}{\gamma} \frac{T}{p} \frac{\partial p}{\partial t}-u \frac{\partial T}{\partial x}+\frac{\gamma-1}{\gamma} k \frac{T}{p}\left(\frac{\partial^{2} T}{\partial x^{2}}\right)+K\left(T_{H X}-T\right)
$$

where the factor $K$ is the heat transfer coefficient between the heat exchanger and the fluid. Outside of the heat exchanger, $\mathrm{K}$ is set to zero. The pressure and the velocity are imposed assuming both a traveling wave and no spatial variations:

$$
\begin{gathered}
p(t)=p_{0}+p_{1} \sin (\omega t) \\
u(t)=u_{1} \sin (\omega t)
\end{gathered}
$$

The temperature equation is solved with the MATLAB function pdepe(), which solves initialboundary problems for parabolic partial differential equations in one-dimension. The temperature is calculated for five periods and the temperature is averaged over the last period.

\section{Results and discussion}

The results for the two CFD models are discussed separately in the following subsections.

\section{Ideal heat exchanger model}

In this subsection the simulation results of the ideal heat exchanger CFD model are presented. The mean temperature profiles for two simulations are shown in Figure 3 over the dimensionless $x$-coordinate. The blue dotted line shows the mean temperature assuming a heat conductivity of $\kappa=0.152 \mathrm{~W} / \mathrm{mK}$ corresponding to helium. A clear minimum in the mean temperature can be seen within one displacement amplitude of the ideal heat exchanger. Furthermore, at the right of the domain heat is conducted towards the outside of the domain. 
In Figure 3 the green line shows the mean temperature profile with zero heat conductivity. In this case the effect of the ideal heat exchanger only extends within two displacement amplitudes $\xi_{1}$. The black dashed line in Figure 3 shows the analytical solution given in Equation (1). The analytical solution overlays the green line as in both cases no heat conduction is assumed. It can be concluded that the applied boundary condition is correctly implemented and that the boundary can model an ideal heat exchanger. When heat conduction is taken into account in the simulation, the minimum temperature is smoothed out. Heat is conducted into the rest of the domain and the mean temperature profile is influenced beyond two displacements amplitudes $\xi_{1}$. The figure also indicates that additional losses are introduced due to the conduction over the right boundary [2].

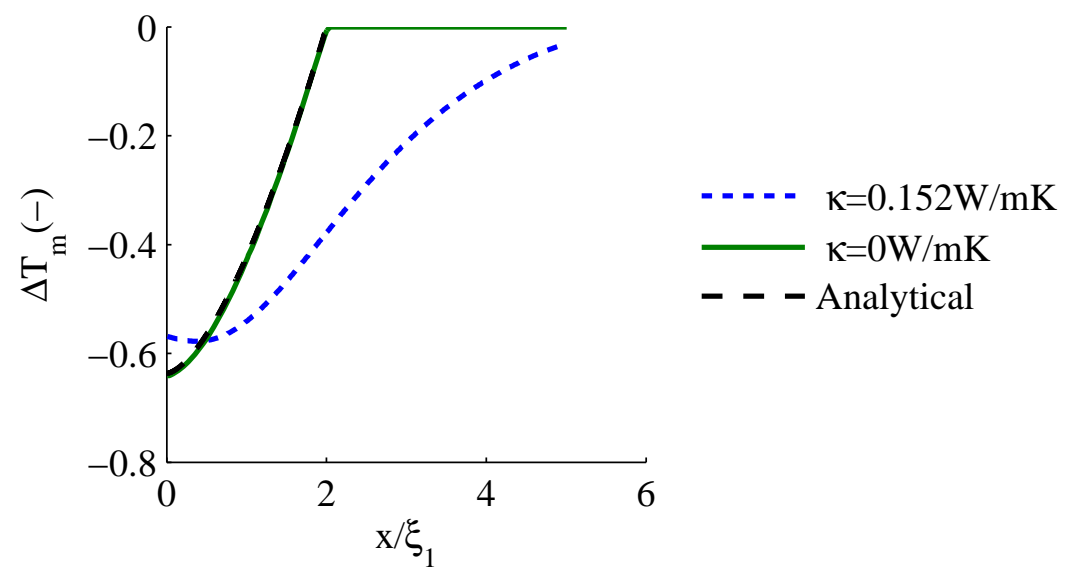

Figure 3: Deviation of the mean temperature normalized with the adiabatic temperature amplitude and plotted over the dimensionless $x$-coordinate.

\section{Stacked screen model}

In this subsection the results for the stacked screen CFD model are discussed. The temperature is averaged over the fifth period and plotted over the dimensionless $x$-coordinate in Figure 4. The two cylinders modeling the stacked screen heat exchanger are located at $x / \xi_{1}=0$. The profile is point symmetric around this point and the entrance effects on both sides of the heat exchanger are modeled. The black dash-dot line shows the numerical solution of the simplified model with a $K$-value chosen to fit the CFD simulation. The two mean temperature profiles are in good agreement with each other. This is also the case with the temperature profiles averaged over the first four periods. In other words, the viscous effects, which are neglected in the simplified model, do not have a large influence on the mean temperature profile at low pressure amplitudes.

\section{Conclusion}

A first step was taken with CFD to investigate the entrance effects near a stacked screen heat exchanger. Two CFD models were presented. From the first model it could be concluded that the dedicated boundary condition, modeling the ideal heat exchanger was correctly implemented and worked well. Furthermore, it is shown that heat conduction flattens the mean temperature profile compared to the case without heat conduction. With the second test case it is shown that solving only the one dimensional heat equation, Equation (2), for a given pressure and velocity gives similar results compared to CFD: i.e. viscous effects do not play an important role at low amplitudes. 
In future work the regenerator model will be extended so that high pressure amplitude simulations can be carried out with various phasing between pressure and the velocity. This will allow the investigation of the influence of vortex generation on the heat transfer in the oscillating flow and provide a better understanding of the heat transfer in a stacked screen heat exchanger.

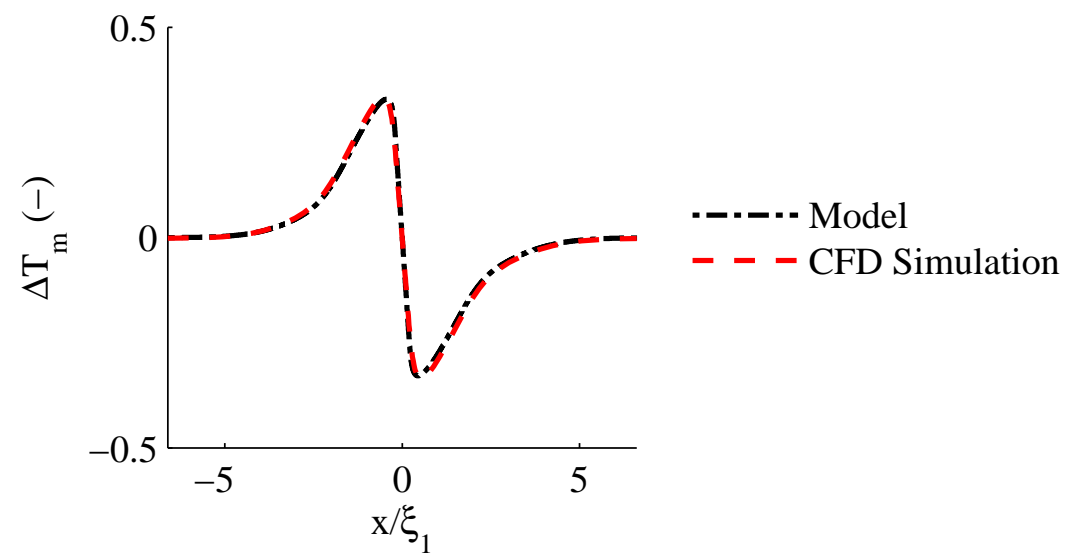

Figure 4: Temperature amplitude averaged over the fifth period normalized with the adiabatic temperature amplitude and plotted over the dimensionless $x$-coordinate.

\section{References}

[1] Backhaus, S. and Swift, G. W.: A thermoacoustic Stirling heat engine. Nature 399, 335-338 (1999)

[2] Berson, A., Poignand, G., Blanc-Benon, P., and Comte-Bellot, G.: Nonlinear temperature Field near the stack ends of a standing-wave thermoacoustic refrigerator. International Journal of Heat and Mass Transfer 54, 4730-4735 (2011)

[3] Gusev, V., Lotton, P., Bailliet, H., Job, S., and Bruneau, M.: Thermal wave harmonics generation in the hydrodynamical heat transport in thermoacoustics. The Journal of the Acoustical Society of America 109, 84-90 (2001)

[4] Kittel, P.: The Temperature Profile within Pulse Tubes. Advances in Cryogenic Engineering 43, 1927-1932 (1998)

[5] Liao, Z.-P.: Extrapolation non-reflecting boundary conditions. Wave Motion 24, 117-138 (1996)

[6] Matveev, K. I., Swift, G. W., and Backhaus, S.: Analytical solution for temperature profiles at the ends of thermal buffer tubes. International Journal of Heat and Mass Transfer 50, 897-901 (2007)

[7] Matveev, K. I., Swift, G. W., and Backhaus, S.: Temperatures near the interface between an ideal heat exchanger and a thermal buffer tube or pulse tube. International Journal of Heat and Mass Transfer 49, 868-878 (2006)

[8] Rott, N.: Thermoacoustics. Advances in Applied Mechanics 20, 135-175 (1980)

[9] Storch, P. J., Radebaugh, R., and Zimmerman, J. E.: Analytical model for the refrigeration power of the orifice pulse tube refrigerator. National Institute of Standards and Technology (U.S.) (1990)

[10] Swift, G. W.: Thermoacoustics: a unifying perspective for some engines and refrigerators. Acoustical Society of America through the American Institute of Physics (2002)

[11] Ward, B., Clark, J., and Swift, G.: Design Environment for Low-Amplitude ThermoAcoustic Energy Conversion (DeltaEC) Users Guide (Version 6.2). Los Alamos National Laboratory (2008)

[12] ANSYS, Inc., ANSYS FLUENT 14.0 User's Guide (2013) 